\title{
(C) OPEN ACCESS \\ SNP located in an AluJb repeat downstream of TMC01, rs4657473, is protective for POAG in African Americans
}

\author{
Lana Verkuil, ${ }^{1}$ Ian Danford, ${ }^{1}$ Maxwell Pistilli, ${ }^{1}$ David W Collins, ${ }^{1,2}$ Harini V Gudiseva, \\ Ben T Trachtman, ${ }^{1}$ Jie He, ${ }^{1}$ Sonika Rathi, ${ }^{1}$ Naqi Haider, ${ }^{1}$ Gui-shuang Ying, ${ }^{3}$ \\ Venkata R M Chavali, ${ }^{1}$ Joan Marie O’Brien ${ }^{1}$
}

\begin{abstract}
- Additional material is published online only. To view please visit the journal online (http://dx.doi.org/10.1136/ bjophthalmol-2018-313086).

${ }^{1}$ Ophthalmology, Scheie Eye Institute, Philadelphia, Pennsylvania, USA

${ }^{2}$ Emeryville, California, USA ${ }^{3}$ Ophthalmology, University of Pennsylvania, Philadelphia, Pennsylvania, USA
\end{abstract}

Correspondence to Dr Venkata R M Chavali, Ophthalmology, Scheie Eye Institute, Philadelphia, PA 19104, USA; vchavali@ pennmedicine.upenn.edu

Received 23 August 2018 Revised 4 January 2019 Accepted 20 February 2019 Published Online First 12 March 2019

\begin{abstract}
Aims To determine the association of single nucleotide polymorphisms (SNPs) downstream from the TMCO1 gene with primary open-angle glaucoma (POAG) in African Americans (AA).

Methods AA subjects were recruited for the Primary Open-Angle African American Glaucoma Genetics (POAAGG) study from the Scheie Eye Institute and its satellite sites in Philadelphia. A region containing an AluJb repeat and seven SNPs, including rs4656461 near the TMCO1 gene, were PCR-Sanger sequenced from POAAGG cases $(n=1537)$ and controls $(n=1570)$. Association between POAG and SNPs near TMCO1 was investigated by logistic regression analysis. Phenotypic trait associations with these SNPs were assessed by analysis of variance. Electrophoretic mobility shift assay (EMSA) was performed to assess the affinity of human T-box 5 (TBX5) protein for a predicted binding motif in the TMCO1 region. Dual Luciferase assays were performed by transfecting recombinant plasmids containing the region surrounding the above SNPs in HEK293T and trabecular meshwork cells.
\end{abstract}

Results The SNP rs4657473 (C>T) was associated with POAG; the TT genotype was protective (OR 0.20, 95\% Cl 0.09 to $0.42 ; p<0.001)$. No significant associations were found between the TMCO1 variants and phenotypic traits. EMSA confirmed the affinity of TBX5 for a predicted binding motif containing TMCO1 SNP rs 4657475. Luciferase assays demonstrated a regulatory function for the genomic region around SNP rs4656561, located within AluJb repeat.

Conclusion Our results demonstrate that a SNP downstream of TMC01, rs4657473, is associated with POAG in an AA population. Our studies suggest a regulatory role for the previously POAG-associated locus near the TMCO1 gene that may affect gene expression.

\section{INTRODUCTION}

Glaucoma is a complex neurodegenerative disease and the leading cause of irreversible blindness worldwide. ${ }^{1}$ Primary open-angle glaucoma (POAG) is a progressive degeneration of retinal ganglion cells and their axons in the optic nerve that leads to characteristic changes in the optic disc and retinal nerve fibre layer (RNFL) and corresponding visual field loss. ${ }^{2}$ Risk factors for POAG include elevated intraocular pressure (IOP), advanced age, family history and African American (AA) race; however, the exact aetiology of POAG remains unknown. ${ }^{3}$ In recent years, family linkage and genome-wide association studies (GWAS) have made increasingly clear the importance of genetic risk factors in the development and progression of glaucoma. ${ }^{4}$

The TMCO1 region has been identified as a likely contributor to the genetic risk for POAG. A single nucleotide polymorphism (SNP), rs4656461, located at the $1 \mathrm{q} 24$ locus in a non-coding region $\sim 6.5 \mathrm{~kb}$ downstream to TMCO1 was linked $\left(\mathrm{p}=6.0 \times 10^{-14}\right)$ to severe POAG-induced visual field loss in a GWAS of a Caucasian cohort. ${ }^{5}$ The same TMCO1 locus was associated with POAG in case-control association studies in Pakistani and Han Chinese patients with high tension glaucoma (HTG). ${ }^{67}$ The SNP rs755523, an intronic variant of TMCO1, has also been associated with HTG in the same Han Chinese population and with high IOP in a GWAS of Caucasians $\left(\mathrm{p}=1.6 \times 10^{-8}\right)$. $^{7}$

Few studies have investigated the genetic risk factors of POAG in AA, despite this population having a higher disease prevalence than other populations. ${ }^{589} \mathrm{~A}$ candidate gene case-control study in AAs (1150 cases and 999 controls) and Ghanaian (West African) subjects (483 cases and 593 controls) was performed for eight SNPs in the TMCO1 region including rs4656461. No significant associations were found for TMCO1 SNPs or other loci that have been previously associated with POAG in populations of non-African ancestry, suggesting that these susceptibility alleles may not be relevant in AAs. ${ }^{10}$

We investigated whether SNP rs4656461 and other SNPs downstream from TMCO1 (in an Alu region) are associated with POAG in a large AA cohort. We also examined the impact of TMCO1 SNPs in this Alu region on predicted TBX5 transcription factor binding and interrogated these for a regulatory role.

\section{METHODS}

\section{Patient recruitment and data collection}

Subjects for our case-control study were recruited from the Scheie Eye Institute, University of Pennsylvania and its research affiliate sites in Philadelphia for the Primary Open-Angle African American Glaucoma Genetics (POAAGG) study. Information regarding POAAGG study patient recruitment and data collection and the baseline demographics of the 2520 subjects enrolled as of March 2014 has 
been previously described. ${ }^{11}$ In brief, subjects over 35 years who self-identified as Black were enrolled. We defined POAG as having an open iridocorneal angle and at least one characteristic glaucomatous optic nerve finding (notching, neuroretinal rim thinning, excavation or a nerve fibre layer defect) that corresponded to a visual field defect. All secondary causes of glaucoma were excluded. Informed consent was obtained from all participating subjects. Approval was obtained from the University of Pennsylvania institutional review board and research was conducted in accordance with the Declaration of Helsinki.

\section{Genotyping and association analysis}

Sanger sequencing was performed on genomic DNA from blood and saliva samples from 1537 AA cases and 1570 controls to genotyp e seven SNPs downstream of TMCO1 (rs11430311, rs4657473, rs4657474, rs4657475, rs116461237, rs61058666 and rs4656461) as previously described. ${ }^{12}$ All the seven candidate SNPs in our study were identified from sequencing around $1 \mathrm{~kb}$ of the intergenic region between TMCO1 and ALDH9A1 surrounding the previously associated POAG GWAS SNP, rs4656461 in both AA cases and controls. Briefly, BigDye Terminator chemistry (ThermoFisher, Waltham, Massachusetts, USA) was used, followed by capillary electrophoresis on an ABI 3130xl genetic analyzer (Applied Biosystems, Foster City, California, USA). Linkage disequilibrium (LD) patterns of the TMCO1 SNPs were plotted using LDlink. ${ }^{13}$

Association between disease status and the genotype frequencies and allele frequencies of the seven SNPs was assessed using logistic regression analysis to calculate ORs with 95\% CI while adjusting for age and sex. Correction for multiple comparisons was performed using a Bonferroni correction, requiring a $\mathrm{p} \leq 0.007$ to be considered as statistically significant after accounting for the testing of seven SNPs. Information regarding the quantitative traits of glaucoma, including central corneal thickness (CCT), IOP, cup-to-disc ratio (CDR), mean deviation (MD), pattern standard deviation (PSD) and RNFL thickness, was collected from the electronic medical records for POAG cases only. We did not collect information about quantitative traits from controls. Associations between each SNP and the quantitative traits among POAG cases were performed using analysis of variance (ANOVA). In these analyses, when measures from left and right eyes were available, averaged values for CCT, maximum-recorded IOP, CDR, MD, PSD and RNFL thickness were calculated and used for statistical comparisons. All statistical analyses were conducted using SAS V.9.4 (SAS Institute, Cary, North Carolina, USA).
Electrophoretic mobility shift assay (EMSA) binding studies

The seven SNPs near TMCO1 from the genotype-phenotype study were queried in RegulomeDB to determine whether any lie within predicted transcription factor binding sites. ${ }^{14}$ Three of the SNPs were predicted to lie within a TBX5 binding motif. EMSA was performed using Biotinylated oligonucleotide probes (online supplementary table 1) and purified recombinant protein of human T-box 5 (TBX5) with C-terminal MYC/ DDK tag (Origene, Rockville, MD; TP316520) and the Odyssey Infrared EMSA kit (LiCor, Lincoln, Nebraska; 829-07910). The binding reactions were assembled according to the manufacturer's protocol. TBX5 protein was incubated with the reaction mixture for $10 \mathrm{~min}$ at $4^{\circ} \mathrm{C}$ followed by incubation with the DNA probes for an additional $30 \mathrm{~min}$. For the competition assay, 2000 -fold excess of unlabeled oligonucleotides was added to the reaction mixture before the addition of the labelled oligonucleotide probe. The DNA-protein complexes were electrophoresed through a TBE gel on an ice bath, visualised with a 1:10 000 dilution of IR dye in PBS and 0.1\% Tween 20 and analysed on an Odyssey infrared fluorescence scanner (LiCor Biosciences, Lincoln, Nebraska, USA).

\section{Cloning of the TMCO1 SNP region and generation of luciferase reporter constructs}

Luciferase reporter constructs were generated by cloning a 3337 bp region of genomic DNA surrounding the TMCO1 SNP region (including rs4656461) from four AA individuals that represented naturally occurring haplotypes (figure 1A). Genomic DNA samples were selected for cloning based on the prior sanger sequencing of POAAGG subjects. Haplotype construct 1_3337 contained wildtype alleles at all seven TMCO1 SNPs (rs11430311, rs4657473, rs4657474, rs4657475, rs116461237, rs61058666 and rs4656461). The haplotypes associated with the SNP rs4657473 variant $(C>T)$, rs116461237 variant $(G>A)$ and SNP rs4656461 variant $(A>G)$ were associated with constructs 2, 3 and 4_3337 respectively. The construct 4_3337 also had variants at SNPs rs11430311, rs4657474 and rs4657475, which are in LD with rs4656461.

PCR amplification was performed using Phusion High-Fidelity DNA polymerase (NEB) and primers containing Acc65I and EcoRV restriction enzyme sites (online supplementary table 2) to generate the 3337 bp sequences containing TMCO1 SNPs. Each PCR construct was ligated into a vector upstream of a minimal promoter (pGL4.23[luc2/minP]; Promega). The resulting plasmids were transfected into 10-beta Competent E. coli (High

\begin{tabular}{|c|c|c|c|c|c|c|c|c|}
\hline $\begin{array}{c}\text { Insert } \\
\text { Number }\end{array}$ & SNP & 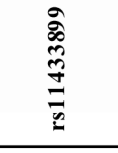 & $\begin{array}{l}\text { R } \\
\frac{1}{5} \\
\text { in } \\
+ \\
0 \\
0\end{array}$ & 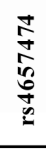 & $\begin{array}{l}\frac{2}{8} \\
\mathbb{1} \\
6 \\
0 \\
0 \\
0 \\
0\end{array}$ & 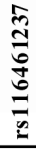 & 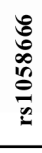 & $\begin{array}{l}5 \\
0 \\
+0 \\
0 \\
0 \\
0 \\
+0\end{array}$ \\
\hline 1 & all wildtype & ins T/insT & $\mathrm{C}$ & $\mathrm{G}$ & $\mathrm{C}$ & $\mathrm{G}$ & $\mathrm{G}$ & A \\
\hline 2 & $\begin{array}{l}\text { rs } 4657473 \\
(\mathrm{C}>\mathrm{T})\end{array}$ & ins T/insT & $\mathrm{T}$ & G & $\mathrm{C}$ & G & G & A \\
\hline 3 & $\begin{array}{l}\text { rs } 116461237 \\
(G>A)\end{array}$ & ins T/insT & $\mathrm{C}$ & G & $\mathrm{C}$ & A & $\mathrm{G}$ & A \\
\hline 4 & $\begin{array}{l}\mathrm{rs} 4656461 \\
\quad(A>G)\end{array}$ & WT/WT & $\mathrm{C}$ & A & $\mathrm{T}$ & G & G & G \\
\hline
\end{tabular}

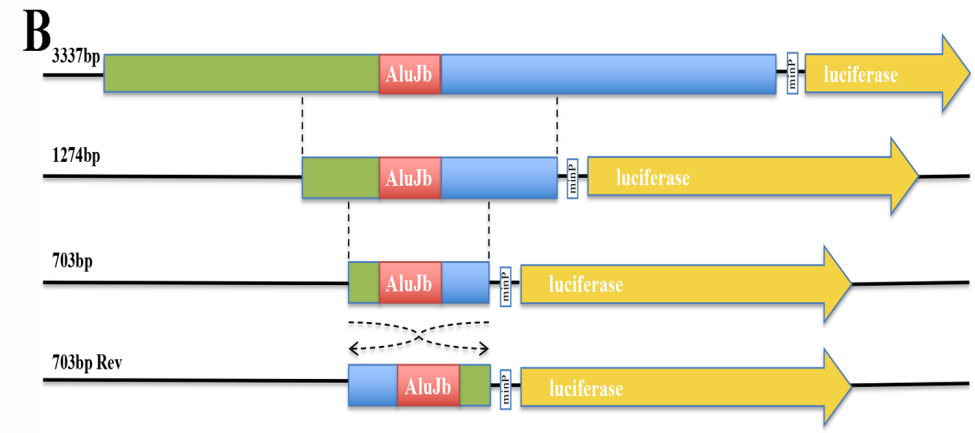

Figure 1 (A) Haplotypes of inserts used in luciferase assay. (B) Luciferase assay was performed with inserts of three sizes surrounding SNP rs4656461 and Alu element. Inserts were formed by serial deletion of the initial 3337 bp construct. 703 bp insert was cloned in both a forward and reverse direction. SNP, single nucleotide polymorphism. 
Efficiency) cells (NEB) and plasmid DNA was quantitated using Qubit Fluorometer 2.0 (ThermoFisher).

In order to identify the minimal regulatory region surrounding the SNPs, serial deletion constructs were developed by selective amplification of the $3337 \mathrm{bp}$ plasmids containing the desired SNPs using primer sets mentioned in online supplementary table 2 to generate $1274 \mathrm{bp}$ and $703 \mathrm{bp}$ constructs, which were again cloned into pGL4.23 vectors (figure 1). The first number for each of the luciferase clones indicates the set of SNPs in the clone (figure 1A) and the second number indicates the size of the clone (figure 1B). All recombinant plasmids were verified by Sanger sequencing. Nearly 300-400 bp of insert sequence surrounding SNP rs4656461 was compared with previous genotyping.

\section{Dual luciferase reporter assay}

Transcriptional regulatory activity of all inserts was interrogated by dual luciferase reporter assay in HEK293T cells (human embryonic kidney ATCC-CRL3216) and primary trabecular meshwork (TM) cells (gift from Dr Markus Kuehn, University of Iowa). To validate the authenticity of the TM cells, we performed the MYOC induction experiment with DEX in the TM cells. We used Passage 2 (P2) to Passage 4 (P4) primary TM cultures for all our experiments. To characterise the TM cultures, we induced 65\%-70\% confluent P4 TM cultures with $100 \mathrm{mM}$ dexamethasone (Sigma) for 3 days. The RNA was isolated from the induced and uninduced TM cultures and was converted to cDNA using Superscript III One-Step RT-PCR kit (Invitrogen, CA) and a quantitative Real-time PCR was performed using primers (5'CATCTGGCTATCTCAGGAGTGG-3'; Rev-5'- TCTCGCAT CCACACACCATAC-3') to amplify Myocilin transcript and the gene expression was compared with GAPDH. We found that there was a 7-fold increase in the Myocilin expression in TM cells induced with Dexamethasone when compared with uninduced controls (online supplementary figure S1).

Both HEK293T and TM cells were plated in a 24-well format and cultured at $37^{\circ} \mathrm{C}$ with $5 \% \mathrm{CO}_{2}$. Cells were seeded on a 96-well white opaque bottomed luminometric plates (Falcon) at $\sim 60 \%$ confluency 24 hours prior to transfection. Cells were transiently transfected with reporter plasmids using Lipofectamine 3000 following manufacturer's instructions (ThermoFisher). Renilla luciferase vector (pGL4.74[hRluc/TK]; Promega) was cotransfected with experimental plasmids to control for transfection efficiency. Luciferase activity was measured following protocol from Pierce Renilla-Firefly Luciferase Dual Assay Kit (Thermo Scientific) on an Infinite 200 PRO plate reader (Tecan). Three replicates were analysed for each transfection set, and the ratio of Firefly luciferase activity to Renilla luciferase activity was used to determine the transfection efficiency. Plasmids were compared by one-way ANOVA and student's t-test for differential luciferase activity. In HEK293T cells, three replicates of 3337 bp and 703 bp Rev inserts were tested in triplicate transfection sets, while 1274 bp and 703 bp inserts were performed as a single transfection set. In TM cells, all inserts were tested in duplicate transfection sets.

\section{RESULTS}

\section{TMCO1 genotype association with glaucoma phenotype}

The present case-control study included 1537 AA POAG cases and 1570 AA controls. Patients with POAG had a mean age of 70.9 years compared with 61.5 years in the controls $(\mathrm{p}<0.001)$. $60.4 \%$ of the POAG cases were female, while $69.4 \%$ of the controls were female $(p<0.001)$. Demographic and clinical characteristics of the cohort are shown in table 1 .
Table 1 Demographic and clinical characteristics of the cohort

\begin{tabular}{lllll}
\hline & \multicolumn{4}{c}{ Glaucoma status } \\
\cline { 2 - 5 } & & Case $(\mathrm{n}=1537)$ & Control $(\mathrm{n}=1570)$ & P value \\
\hline Female, \% & $\mathrm{F}$ & $929(60.4 \%)$ & $1090(69.4 \%)$ & $<0.001$ \\
\hline Age, years & $\mathrm{n}$ & 1537 & 1569 & $<0.001$ \\
& Mean (SD) & $70.9(11.3)$ & $61.5(11.8)$ & \\
Maximum-recorded & $\mathrm{n}$ & 1535 & 1515 & $<0.001$ \\
IOP (mean), mm Hg & Mean (SD) & $25.9(8.0)$ & $17.1(3.2)$ & \\
CDR (mean) & $\mathrm{n}$ & 1494 & 1247 & $<0.001$ \\
& Mean (SD) & $0.71(0.16)$ & $0.33(0.13)$ & \\
CCT (mean) & $\mathrm{n}$ & 1470 & - & - \\
& $\mathrm{Mean}(\mathrm{SD})$ & $531.0(38.8)$ & - & - \\
RNFL (mean) & $\mathrm{n}$ & 1096 & - & - \\
& Mean (SD) & $72.9(13.3)$ & - & - \\
PSD (mean) & $\mathrm{n}$ & 1106 & - & \\
& Mean (SD) & $5.1(3.1)$ & - & \\
MD (mean) & $\mathrm{n}$ & 1106 & - & \\
& Mean (SD) & $-7.4(7.8)$ & - & - \\
\hline
\end{tabular}

$\mathrm{CCT}$, central corneal thickness; CDR, cup-to-disc ratio; IOP, intraocular pressure; $\mathrm{MD}$, mean deviation; PSD, pattern standard deviation; RNFL, retinal nerve fibre layer.

Since our cohort enrolment was done by self-reporting as Black, we have performed a PCA of the cases and controls from our study to assess our cohorts for outliers in heredity. Boxplots results showing the comparison of the PC1 score between the cases and controls showed no statistically significant difference $(\mathrm{p}=0.06)$ (online supplementary figure S2). Genotyping efficiency was $\geq 99.8 \%$ for the seven SNPs. LD analysis revealed that SNPs rs11430311, rs4657474 and rs4657475 are in LD with SNP rs4656461 (figure 2B). Genotype frequencies and $p$ values for the association of seven TMCO1 SNPs in POAG cases and controls are shown in table 2.

We observed a significant association of POAG with SNP rs4657473, with a protective effect shown by the TT variant at this locus (OR $0.20,95 \%$ CI 0.09 to $0.42 ; \mathrm{p}<0.001$ ). The allele association analysis found no statistically significant association between any of the seven SNPs with POAG (table 2). The Hardy-Weinberg (HW) equilibrium $\mathrm{p}$ values, and the logistic regression $\mathrm{p}$ values for the allele frequency are also shown in table 2. Interestingly, the rs4657473 SNP is out of HW equilibrium, but the other SNPs are agreeing with it. These results indicate that the population itself is not at disequilibrium, but rather that this SNP may be a specific newer mutation, which is not inconsistent with it being associated with case status. The $\mathrm{p}$ value for the trend is also not significant, this is explained because the CT group is almost identical to the CC group, suggesting that only that the homozygous TT is necessary to be associated with the disease. No significant association was found for SNP rs4656461 or for any of the seven TMCO1 SNPs with the quantitative traits of POAG among the case subjects (online supplementary table 3).

\section{EMSA binding studies}

EMSA studies were performed to assess the binding of transcription factor TBX5 to its predicted binding motif encompassing 3 TMCO1 SNPs, rs4657475 (in LD with rs4656461), rs116461237 and rs61058666. EMSA showed that TBX5 formed complexes with the probes containing the three wildtype TMCO1 SNPs (figure 3). Introduction of variant rs116461237 (G>A) disrupted TBX5 binding. However, variants at rs4657475 and rs6105866 did not interfere with complex formation even when random 
A

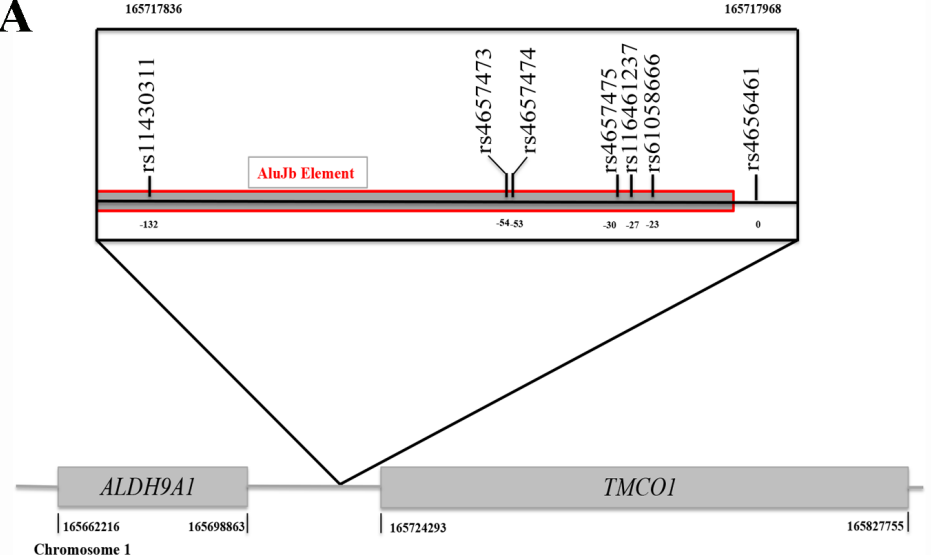

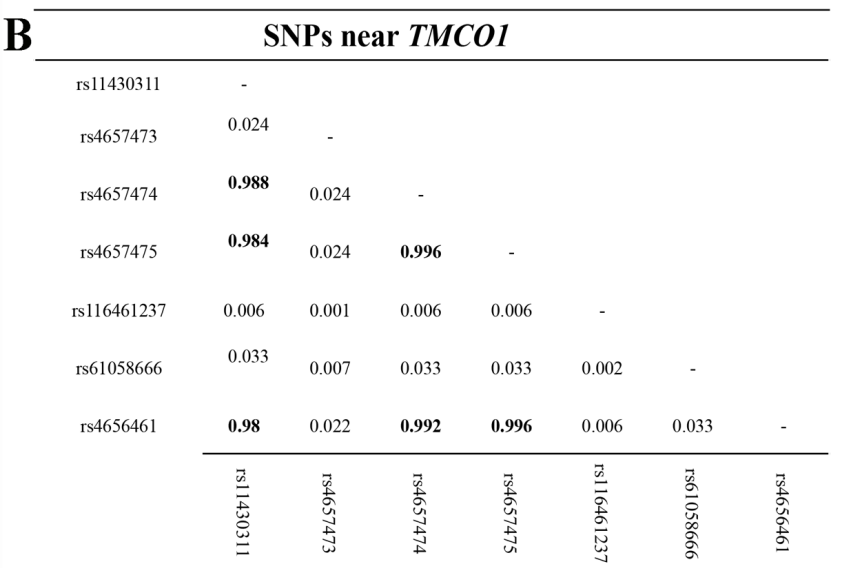

Figure 2 (A) Schematic showing POAG-associated SNP rs4656461 and six SNPs in an adjacent Alu element lie in an intergenic region between genes TMCO1 and ALDH9A1. (B) LD relationships are shown for TMCO1 SNPs. SNP rs4656461 is in LD with rs11430311, rs4657474 and rs4657475. LD, linkage disequilibrium; SNP, single nucleotide polymorphism.

alleles were introduced at these positions. Notably, TBX5 did not bind to the Alu consensus repeat motif, indicating that the affinity of this Alu region for TBX5 is an acquired trait.

\section{Regulatory role of the TMCO1 SNP region}

To test for a regulatory effect of SNP rs4656461 and its adjacent Alu region on gene transcription, a 3337 bp region surrounding SNP rs4656461 was cloned into a pGL4-derived vector containing a firefly luciferase reporter gene under the presence of a minimal promoter. Sequencing analysis revealed that Insert 2_703 had acquired the minor allele variant for SNP rs11430311, which was not in the expected haplotype. Sequence analysis of three inserts of all sizes (3_3337, 3_1274, 3_703 and 3_703Rev) also contained an undesired mutation $201 \overline{\mathrm{b}} \mathrm{p}$ upstream of SNP rs11430311 (online supplementary figure 3).to

In HEK293T cells, inserts 1_3337, 2_3337, 3_3337 and 4_3337 repressed luciferase expression when compared to the empty pGL4.23 vector. Repression was significantly greater for Insert 2_3337 when compared to both Inserts 3_3337 ( $p=0.03)$ and 4_3337 ( $\mathrm{p}=0.04)$ (figure 4A). Serial deletion of $3337 \mathrm{bp}$ constructs demonstrated increasing levels of luciferase repression with $1274 \mathrm{bp}$ and 703 bp constructs. ANOVA results comparing luciferase expression between the different sized inserts within each haplotype were all significant (Insert $1 \mathrm{p}<0.0001$, Insert 2 $p=0.0006$, Insert $3 p=0.003$, Insert $4 p<0.0001$ ) (figure 4B). In the reverse orientation, the $703 \mathrm{bp}$ constructs showed enhancer activity, with Insert 4_703Rev showing significantly more reporter gene expression than wildtype Insert 1 703Rev $(p=0.03)$ (figure 4C).

In primary TM cells, 3337 bp inserts did not have a regulatory effect on luciferase expression, while $1274 \mathrm{bp}$ and 703 bp constructs demonstrated luciferase repression (online supplementary figure 4). Only one significant difference in repressor activity was found, which was between Insert 1_1274 and 3_1274. Serial deletion of the inserts did not demonstrate the same pattern of core repressor activity as in HEK293T cells, and changing the orientation of the $703 \mathrm{bp}$ inserts did not result in enhancer activity .(online supplementary figure 5).

\section{DISCUSSION}

The SNPs downstream from and within TMCO1 have been implicated in the genetic risk of POAG. The TMCO1 SNP rs4656461 was associated with POAG in Caucasians and then later in Asian populations with high IOP. ${ }^{715}$ Fewer studies have investigated the role of this variant in populations of African ancestry. ${ }^{16}$ The association of SNP rs4656461 with POAG was not replicated in our AA cohort. Our results are consistent with a prior case-control study, in which SNPs associated with POAG in populations of non-African ancestry (including SNP rs4656461) had no significance in those of African descent. ${ }^{10}$ However, we report a novel association between POAG and SNP rs4657473 (located within $\sim 50 \mathrm{bp}$ of rs4656461). Those with the homozygous TT genotype are at a lower risk of developing POAG. The allele frequencies of the SNP rs4657473 variant $(C>T)$ are lower in Africans (7\%) and African-Americans (15\%) compared with European populations (25\%) (1000Genomes). If the TT genotype is indeed protective, perhaps this lower prevalence contributes in some part to the greater prevalence of POAG in those of African ancestry. ${ }^{1}$ An additional association between a SNP in the TMCO1 region with POAG further strengthens the evidence that this region is involved in glaucoma pathogenesis. TMCO1 SNPs have previously been associated with high IOP and younger age at diagnosis. ${ }^{7} 1517$ However, no associations were found between TMCO1 SNPs and quantitative glaucoma parameters such as IOP, CCT, CDR or RNFL thickness in our cohort and did not appear to define a glaucoma subtype or increase severity of disease.

It has largely been assumed that SNP rs4656461 is the functional POAG SNP and that it acts on its most proximal gene, TMCO1, that encodes a highly conserved transmembrane protein with a coiled-coil domain. ${ }^{18} 19$ However, the pathway through which rs4656461 is involved in POAG pathogenesis remains to be known. Recently, Wang et al described TMCO1 as an endoplasmic reticulum (ER) transmembrane protein that prevents ER calcium ion $\left(\mathrm{Ca}^{2+}\right)$ stores from overfilling by forming a $\mathrm{Ca}^{2+}$-selective ion channel in response to $\mathrm{Ca}^{2+}$ overloading. ${ }^{18}$ TMCO1 protein expression has been found in several types of human ocular tissues, including the iris, ciliary body, retina and optic nerve. $^{15}$ The mRNA expression levels of TMCO1 in human ocular tissue were shown to be highest in the TM and retina. ${ }^{17}$ TMCO1 protein may play a role in tumour suppression and/or cell cycle regulation, disrupting cellular functions and causing oxidative stress in ocular tissues. ${ }^{415}$

The intergenic region containing POAG-associated TMCO1 SNPs harbours an Alu element. Alu sequences belong to the Short Interspersed Element (SINE) family of human repetitive 
Table 2 Genotype frequencies for 7 TMCO1 SNPs and association with POAG

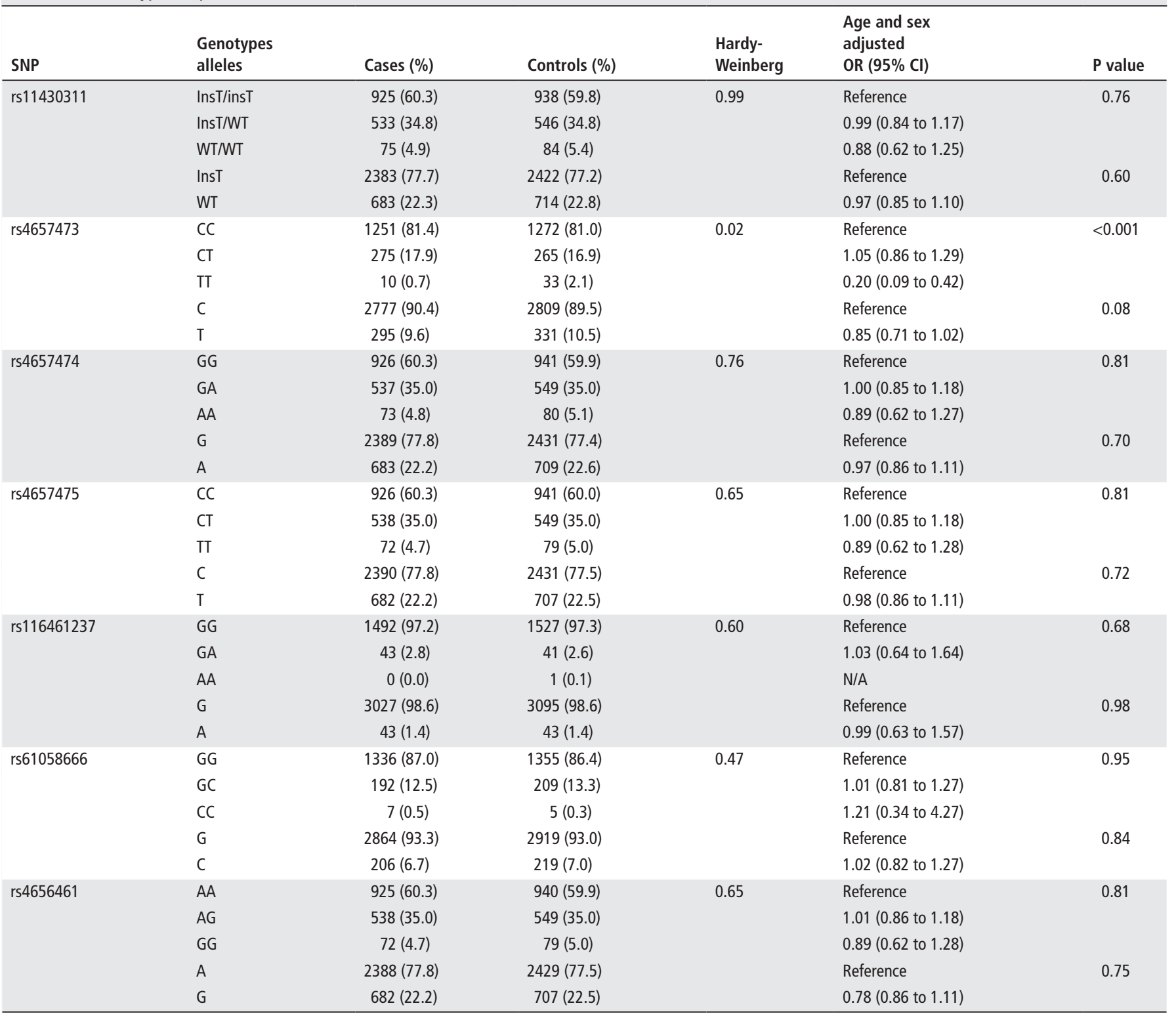

${ }^{*} \mathrm{P}$ values for any difference between three genotypes, and linear difference by number of alleles, respectively.

POAG, primary open-angle glaucoma; SNP, single nucleotide polymorphism.

DNA elements. ${ }^{20}$ They are commonly located in introns, $3^{\prime}$ untranslated gene regions and intergenic genomic regions. ${ }^{20} \mathrm{Alu}$ elements are transposable and after insertion into a new region of the genome acquire random mutations over time. ${ }^{20}$ The $A l u$ element adjacent to SNP rs4656461 is from the AluJb subfamily, one of the oldest subfamilies, dating from 80 to 100 million years ago. ${ }^{21}$ Alus tend to be located near and within genes, which may be due to positive selection exerted as a result of functional roles, such as regulation of gene transcription. ${ }^{21}$ Alus have been shown to act as enhancers or silencers. ${ }^{22}$ They have an ability to introduce new TF binding sites, promote nucleosome assembly, induce methylation or form secondary structures. ${ }^{20} 22$ Alu consensus sequences were shown to be enriched for transcription factor binding motifs, including those involved in pathways of eye, brain and central nervous system development. ${ }^{21}$ The influence on POAG risk of TMCO1 SNPs may be due to the presence of an Alu sequence and the SNPs it contains, which could modulate differential transcriptional regulation and variable transcription factor binding.

To investigate a possible regulatory role of TMCO1 SNPs in this $A l u$ region, we interrogated their transcription factor binding potential. A RegulomeDB query predicted that TMCO1 SNPs rs4657475, rs116461237 and rs61058666 are located in a TBX5 binding motif (figure 3A). TBX5 is a member of the T-box transcription factor gene family, whose genes regulate many developmental processes in vertebrates and invertebrates. ${ }^{23}$ TBX5 has been implicated in the dorsal-ventral patterning of the eye and the establishment of visual projections between the retina and tectum. ${ }^{24}$ Using EMSA, we confirmed that TBX5 forms complexes with an oligonucleotide sequence containing this predicted DNA binding motif, encompassing the three wildtype TMCO1 SNPs. The SNP variant rs116461237 disrupted complex formation, suggesting that variants at this locus may lead to differential transcription factor binding and thereby 
A

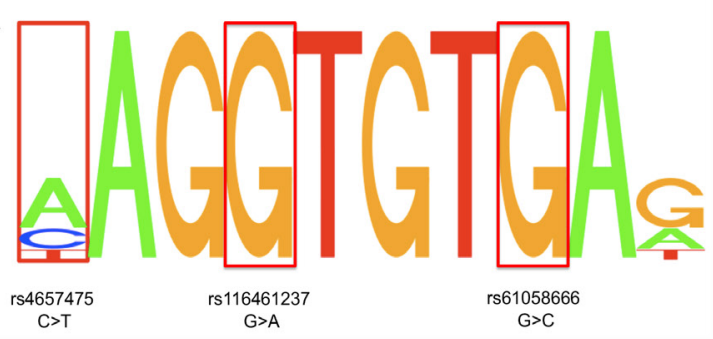

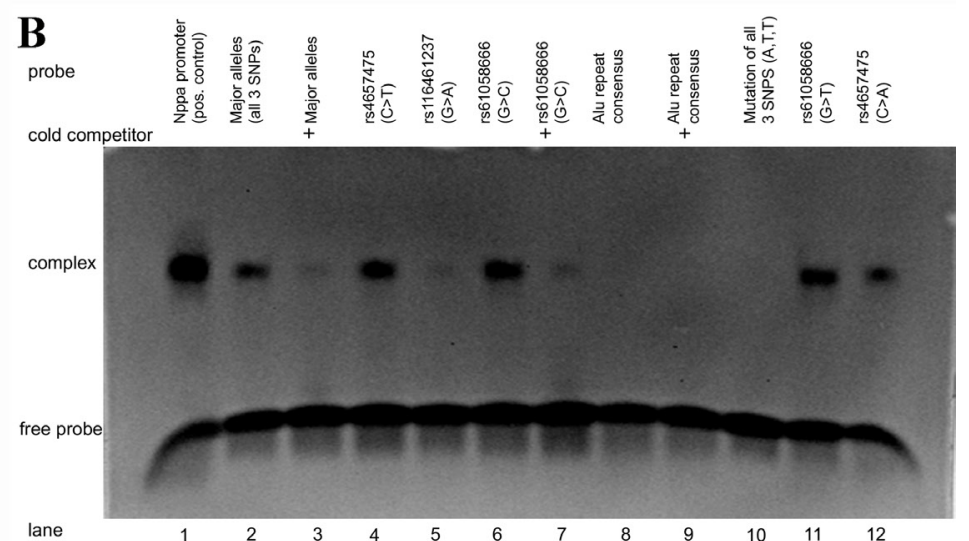

Figure 3 (A) Three TMCO1 SNPs fall within a predicted TBX5 binding motif. (B) EMSA result for variants rs4657475, rs116461237 and rs61058666. Lane 1, Nppa promoter is known to bind to TBX5. Lane 2, wildtype SNP sequence forms complex with TBX5. Lanes 4-7, SNP rs116461237 variant disrupts TBX5 binding. Lanes 8-9, TBX5 does not form DNA-protein complex with Alu consensus sequence. Lanes 10-12, mutation of rs 116461237 only disrupts TBX5-probe complex formation. Lanes 3, 7 and 9 contain cold competitor in addition to probe sequence. SNP, single nucleotide polymorphism.

affect gene regulation. Although the SNP rs116461237 is not associated with POAG in our cohort, we infer that the previously associated SNP rs4656561 along with the LD-associated SNPs rs4657474 and rs4657475 (figure 2B) do not influence TBX5 binding (figure $3 \mathrm{~B}$ ). ${ }^{5}$ Using EMSA, we demonstrated that the $A l u J b$ sequence downstream from TMCO1 has affinity for TBX5, whereas the ancestral Alu sequence did not. This indicates that the TBX5-binding is a new trait, consistent with the proposal that this particular repetitive element has acquired a regulatory function.

Luciferase assays in HEK293T cells demonstrated that the TMCO1 SNP region is able to act as a transcriptional repressor in vitro. Serial deletion of this region revealed a core repressor element of $703 \mathrm{bp}$ surrounding the AluJb sequence. As serial deletions removed the non-Alu parts of the sequence, it appears that the $A l u$ region effected the gene repression. Alu sequences have been shown previously to silence gene transcription. ${ }^{25} 26$ They have also been shown to act as either negative or positive regulators of transcription based on their orientation. ${ }^{27}$ In our study, we confirmed this relationship between Alu orientation and differential effects on gene transcription. The $703 \mathrm{bp}$ sequence when cloned into our reporter vector in the reverse orientation acted to enhance gene expression. The variying levels of repression and/or enhancer activity by the minimal regulatory region in our study need additional studies to establish if these AluJb haplotypes can modulate gene transcription.

When we tested our luciferase reporter constructs in an ocular cell line, primary TM cells, we did not observe the same core repressor pattern or orientation dependent regulatory activity. A different set of transcription factors may be expressed in TM than in HEK293T cells and perhaps TMCO1 SNPs are not relevant in the TM but rather a different ocular cell type. Further testing of other ocular tissues is required. In the luciferase reporter study, we compared the TMCO1 SNP region of interest to an empty vector rather than one with a similar sized random sequence. It has been demonstrated that luciferase reporter activity is inversely correlated with construct size. ${ }^{28}$ However, we do not believe that this confounded our results because we saw
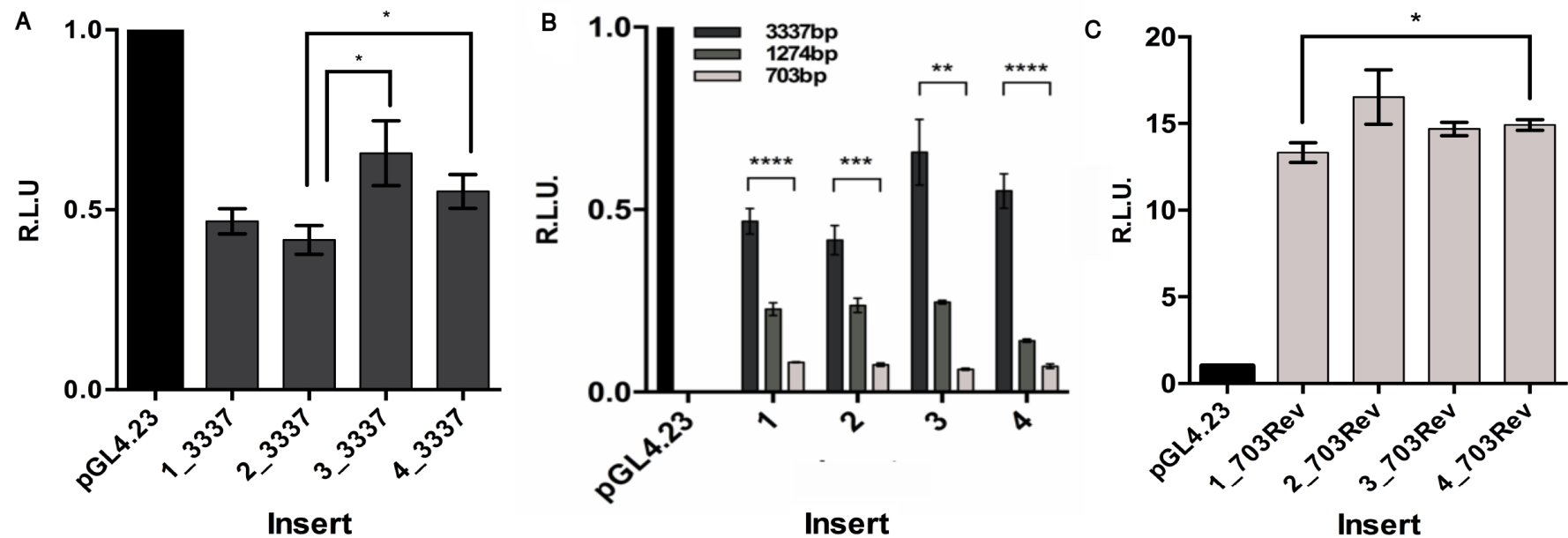

Figure 4 Luciferase assay in HEK293T Cells. (A) 3337 bp inserts repress luciferase expression. Insert with haplotype 2 (rs4657473 C>T) shows greater repression than Inserts 3 ( $r s 116461237 \mathrm{G}>A)(p=0.0261)$ and 4 ( $r s 4656461 A>G)(p=0.0443)$. (B) Serial deletion of 3337 bp inserts identifies the core repressor 703 bp fragment surrounding SNP rs4656461. One-way ANOVA: Insert 1 ( $p<0.0001)$, Insert 2 ( $p=0.006)$, Insert 3 ( $p=0.0028)$, Insert 4 ( $p<0.001)$. (C) 703 bp insert acts as an enhancer when cloned in the reverse orientation. Greater enhancer activity is shown by Insert $4 \_703$ Rev (rs4656461 A>G) over wildtype sequence in Insert 1_703Rev ( $p=0.0252$ ). SNP, single nucleotide polymorphism. 
decreased luciferase expression with serial deletion (shortening) of our construct and saw both enhancement and repression of the $703 \mathrm{bp}$ construct depending on its orientation without a change in its size.

If this $A l u$ region is indeed regulating the transcription of TMCO1, it still remains to be shown that the TMCO1 protein is involved in POAG pathogenesis. In the TMCO1 defect syndrome, a distinct combination of craniofacial dysmorphism, skeletal anomalies and mental retardation are observed. ${ }^{29}$ There have been no eye-related abnormalities reported other than strabismus. ${ }^{29}$ However, the recently described function of TMCO1 as a regulator of cellular calcium homeostasis suggests a mechanism for its involvement in POAG. ${ }^{30}$ Dysregulation of cellular $\mathrm{Ca}^{2+}$ homeostasis, ER stress and $\mathrm{Ca}^{2+}$ overload have been implicated in the pathogenesis of neurodegenerative disorders such as Alzheimer's disease, Parkinson's disease, Huntington's disease and amyotrophic lateral sclerosis. ${ }^{31}$ As suggested in these diseases, it is possible to speculate that perturbation of $\mathrm{Ca}^{2+}$ homeostasis through TMCO1 dysfunction could contribute to the degeneration of retinal ganglion cells in the optic nerve observed in POAG. Alterations in calcium flux may affect aqueous outflow pathways through TM cell volume regulation, possibly explaining why TMCO1 variants have been associated with high $\mathrm{IOP}^{32}$ Alternatively, it is possible that these SNPs may influence the activity of the downstream gene, $A L D H 9 A 1$, which has been implicated as a regulator of IOP by eQTL analaysis. ${ }^{33}$

In conclusion, this study supports the involvement of genetic variation at this TMCO1 SNP locus in the genetic risk of POAG in an AA population and suggests a possible regulatory role for this region. Future studies, however, are needed to determine if this gene region regulates the TMCO1 gene in vivo, and knockout TMCO1 in other ocular cell lines and in animal models are planned to determine the precise role of TMCO1 in the eye.

Contributors LV, VRMC, MP, JMO and G-sY designed the study. LV, SR, NH, ID, JH, HVG, BTT and DWC performed experiments and interpreted data. LV, VRMC, G-sY, $\mathrm{MP}$ and JMO wrote the manuscript.

Funding This work was supported by the National Eye Institute, Bethesda, Maryland (grant \#1R01EY023557-01) and the Department of Ophthalmology at the Perelman School of Medicine, University of Pennsylvania, Philadelphia, PA. Funds also come from the F.M. Kirby Foundation, Research to Prevent Blindness, The Paul and Evanina Bell Mackall Foundation Trust and the National Eye Institute, National Institutes of Health, Department of Health and Human Services, under eyeGENETM and contract Nos. HHSN260220700001C and HHSN263201200001C.

Disclaimer The sponsor or funding organisation had no role in the design or conduct of this research.

Competing interests None declared.

Patient consent for publication Not required.

Provenance and peer review Not commissioned; externally peer reviewed.

Open access This is an open access article distributed in accordance with the Creative Commons Attribution Non Commercial (CC BY-NC 4.0) license, which permits others to distribute, remix, adapt, build upon this work non-commercially, and license their derivative works on different terms, provided the original work is properly cited, appropriate credit is given, any changes made indicated, and the use is non-commercial. See: http://creativecommons.org/licenses/by-nc/4.0/.

\section{REFERENCES}

1 Tham Y-C, Li X, Wong TY, et al. Global prevalence of glaucoma and projections of glaucoma burden through 2040: a systematic review and meta-analysis. Ophthalmology 2014;121:2081-90.

2 Weinreb RN, Khaw PT. Primary open-angle glaucoma. Lancet 2004;363:1711-20.

3 Allingham RR, Liu Y, Rhee DJ. The genetics of primary open-angle glaucoma: a review. Exp Eye Res 2009;88:837-44.
4 Janssen SF, Gorgels TGMF, Ramdas WD, et al. The vast complexity of primary open angle glaucoma: disease genes, risks, molecular mechanisms and pathobiology. Prog Retin Eye Res 2013;37:31-67.

5 Burdon KP, Macgregor S, Hewitt AW, et al. Genome-wide association study identifies susceptibility loci for open angle glaucoma at TMCO1 and CDKN2B-AS1. Nat Genet 2011;:43:574-8.

6 Micheal S, Ayub H, Khan Ml, et al. Association of known common genetic variants with primary open angle, primary angle closure, and pseudoexfoliation glaucoma in Pakistani cohorts. Mol Vis 2014;20:1471-9.

7 Chen $Y$, Hughes $G$, Chen X, et al. Genetic variants associated with different risks for high tension glaucoma and normal tension glaucoma in a Chinese population. Invest Ophthalmol Vis Sci 2015;56:2595-600.

8 Danford ID, Verkuil LD, Choi DJ, et al. Characterizing the "POAGome": A bioinformatics-driven approach to primary open-angle glaucoma. Prog Retin Eye Res 2017:58:89-114

9 Kapetanakis VV, Chan MPY, Foster PJ, et al. Global variations and time trends in the prevalence of primary open angle glaucoma (POAG): a systematic review and metaanalysis. Br J Ophthalmol 2016;100:86-93.

10 Liu Y, Hauser MA, Akafo SK, et al. Investigation of known genetic risk factors for primary open angle glaucoma in two populations of African ancestry. Invest Ophthalmol Vis Sci 2013;54:6248-54.

11 Charlson ES, Sankar PS, Miller-Ellis E, et al. The primary open-angle African American glaucoma genetics study: baseline demographics. Ophthalmology 2015;122:711-20.

12 Gudiseva HV, Hansen M, Gutierrez L, et al. Saliva DNA quality and genotyping efficiency in a predominantly elderly population. BMC Med Genomics 2016;9.

13 Machiela MJ, Chanock SJ. LDlink: a web-based application for exploring populationspecific haplotype structure and linking correlated alleles of possible functional variants. Bioinformatics 2015;31:3555-7.

14 Boyle AP, Hong EL, Hariharan M, et al. Annotation of functional variation in personal genomes using RegulomeDB. Genome Res 2012;22:1790-7.

15 Sharma S, Burdon KP, Chidlow G, et al. Association of genetic variants in the TMCO1 gene with clinical parameters related to glaucoma and characterization of the protein in the eye. Invest Ophthalmo/ Vis Sci 2012:53:4917-25.

16 Scheetz TE, Faga B, Ortega L, et al. Glaucoma risk alleles in the ocular hypertension Treatment study. Ophthalmology 2016;123:2527-36.

17 van Koolwijk LME, Ramdas WD, Ikram MK, et al. Common genetic determinants of intraocular pressure and primary open-angle glaucoma. PLOS Genet 2012;8:e1002611.

18 Wang Q-C, Zheng Q, Tan $\mathrm{H}$, et al. TMCO 1 Is an ER Ca(2+) Load-Activated $\mathrm{Ca}(2+)$ Channel. Cell 2016;165:1454-66.

19 Zhang Z, Mo D, Cong P, et al. Molecular cloning, expression patterns and subcellular localization of porcine TMC01 gene. Mol Biol Rep 2010;37:1611-8.

20 Batzer MA, Deininger PL. Alu repeats and human genomic diversity. Nat Rev Genet 2002:3:370-9.

21 Polak P, Domany E. Alu elements contain many binding sites for transcription factors and may play a role in regulation of developmental processes. BMC Genomics 2006;7.

22 Rowold DJ, Herrera RJ. Alu elements and the human genome. Genetica 2000;108:57-72

23 Naiche LA, Harrelson Z, Kelly RG, et al. T-box genes in vertebrate development. Annu Rev Genet 2005;39:219-39.

24 Leconte L, Lecoin L, Martin P, et al. Pax6 interacts with cVax and Tbx5 to establish the dorsoventral boundary of the developing eye. J Biol Chem 2004;279:47272-7.

25 Ebihara M, Ohba H, Ohno S-ich, et al. Genomic organization and promoter analysis of the human nicotinic acetylcholine receptor alpha6 subunit (CHNRA6) gene: Alu and other elements direct transcriptional repression. Gene 2002;298:101-8.

26 Sharan C, Hamilton NM, Parl AK, et al. Identification and characterization of transcriptional silencer upstream of the human BRCA2 gene. Biochem Biophys Res Commun 1999:265:285-90.

27 Jacobsen BM, Jambal P, Schittone SA, et al. Alu repeats in promoters are positiondependent co-response elements (coRE) that enhance or repress transcription by dimeric and monomeric progesterone receptors. Mol Endocrinol 2009;23:989-1000.

28 Yin W, Xiang P, Li Q. Investigations of the effect of DNA size in transient transfection assay using dual luciferase system. Anal Biochem 2005;346:289-94.

29 Xin B, Puffenberger EG, Turben S, et al. Homozygous frameshift mutation in TMC01 causes a syndrome with Craniofacial dysmorphism, skeletal anomalies, and mental retardation. Proc Natl Acad Sci U S A 2010;107:258-63.

30 La Rovere RML, Roest G, Bultynck G, et al. Intracellular $\mathrm{Ca}(2+)$ signaling and $\mathrm{Ca}(2+)$ microdomains in the control of cell survival, apoptosis and autophagy. Cell Calcium 2016;60:74-87.

31 Mattson MP. Calcium and neurodegeneration. Aging Cell 2007;6:337-50.

32 Wiederholt M, Thieme H, Stumpff F. The regulation of trabecular meshwork and ciliary muscle contractility. Prog Retin Eye Res 2000;19:271-95.

33 Hysi PG, Cheng C-Y, Springelkamp H, et al. Genome-wide analysis of multi-ancestry cohorts identifies new loci influencing intraocular pressure and susceptibility to glaucoma. Nat Genet 2014;46:1126-30. 